\section{Lymph node yield as a prognostic factor in clinically node negative oral cavity squamous cell carcinoma}

\author{
Abmad A. AlTuwaijri, MBBS, SBORL, \\ Mohammed A. Alessa, MBBS, FRCSC, \\ Alanoud A. Abuhaimed, MBBS, \\ Reenad H. Bedaiwi, MBBS, \\ Mohammad A. Almayouf, MBBS, FRCSC, \\ Majed M. Albarrak, MBBS, FRCSC, \\ Saleh F. Aldhahri, MBBS, FRCSC, \\ Khalid H. Al-Qabtani, MBBS, FRCSC.
}

\section{ABSTRACT}

Objectives: To determine the prognostic significance of nodal yield in patients with clinically node-negative (cN0) oral cavity squamous cell carcinoma (OCSCC).

Methods: This retrospective observational study included 40 patients with cN0 OCSCC who underwent treatment with at least 6 months of follow-up data from November 2012 to April 2020. We recorded the variables, including patient demographics, cancer site, tumor-node-metastasis (TNM) staging, type of treatment, lymph node yield (LNY), histopathologic diagnosis, and recurrence. The recorded data were analyzed with descriptive and interferential statistics using specific tests.

Results: Our study cohort comprised of 27 males and 13 females with a mean age of $60.08_{ \pm} 13.153$ years. Tongue $(55 \%)$ was the commonly affected site. Seventeen (42.5\%) patients belonged to TNM stage II. The mean LNY in our study was $38.65 \pm 25.41$ (range 7-98). Following surgery, 19 (47.5\%) patients further received adjuvant therapies. Recurrence was reported only in $4(10 \%)$ patients. There was no significant difference between LNY and recurrence rate $(p=0.892)$. Factors including, age $(p=0.121)$, gender $(p=0.209)$, site $(p=0.519)$, size of tumor $(p=0.416)$ did not influence the LNY.

Conclusion: There is no correlation between LNY and recurrence in cNO OCSCC patients in our study. Meticulous neck dissection and thorough pathologic reporting prevents TNM under staging and improves the overall survival and prognosis.

Keywords: lymph node yield, oral cavity squamous cell carcinoma, recurrence, selective neck dissection, cN0

Saudi Med J 2021; Vol. 42 (12): 1357-1361 doi: 10.15537/smj.2021.42.12.20210572
$\mathrm{O}$ ral cavity squamous cell carcinoma (OCSCC) accounts for $2 \%$ of all cancers, with an annual incidence of over 300,000 cases worldwide. ${ }^{1}$ Factors including, age, gender, the primary site of the tumor, depth of invasion, lymph node (LN) involvement are considered independent prognostic indicators of OCSCC. ${ }^{2}$ Among these, cervical lymphadenopathy is the most important prognostic indicator of OCSCC; however, the cervical metastasis differs among different oral cavity sites and the clinical stage of the tumor, due to differences in the lymphatic drainage. Currently, surgical resection of the tumor along with selective neck dissection (SND) is the standard treatment of choice for clinically node-negative ( $\mathrm{cN} 0$ ) tumors if the risk of occult nodal disease of the neck is $\geq 20 \%$. ${ }^{3}$

The total number of LNs retrieved during the neck dissection constitutes the lymph node yield (LNY). Previous studies have reported an improved prognosis and survival with higher LNY as it increases the probability of resecting the potential tissue with the occult disease. ${ }^{4,5}$ However, there is a lack of definitive results suggesting an association between higher LNY in different head and neck cancer variants and improved oncologic outcome. Lack of specific guidelines on the extent of neck dissection from SND, minimum LNY values required to eliminate the risk of occult disease attribute to the variable clinical outcome. Moreover, differences in the techniques and protocol followed by the surgeons and pathologists in different institutions also play a role. ${ }^{6}$ This study aimed to determine the prognostic significance of nodal yield in patients with cNO OCSCC.

Methods. We conducted an analytical retrospective observational study in Otolaryngology, Head and Neck Department at King Fahad Medical City, Riyadh, Saudi Arabia. After obtaining clearance from the institutional ethics committee (ref no.: 21-167 dated 26.04.2021), we screened the Institution's electronic medical records of all patients treated for OCSCC from 2012 to 2020. We excluded all patients with clinically positive nodes during tumor-node-metastasis (TNM) staging (8th edition) and other head and neck malignancies. We included a total of 40 patients with TNM staging (clinical and radiological) without nodal involvement

Disclosure. Authors have no conflict of interests, and the work was not supported or funded by any drug company. 
(cN0) who underwent neck dissection with at least 6 months follow-up data for the final analysis.

We recorded the following details: the patient demographics (age, gender), site of cancer, TNM staging based on clinical and radiographic findings, ${ }^{7}$ types of treatment, number of LNs dissected, histopathologic diagnosis, and recurrence. Considering the cN0 staging, all patients underwent supramylohyoid neck dissection (level I to level III). The number of LNs extracted and counted by the pathologist was termed as LNY. The reappearance of cancer within 6 months following treatment was considered persistent disease or treatment failure, while reappearance beyond 6 months was considered a recurrence.

Statistical analysis. The recorded data entered in Microsoft excel was imported to SPSS version 21 (IBMCorp, Armonk, NY, USA) for statistical analysis. Continuous variables were expressed as mean and standard deviations, and categorical variables were expressed as frequency and percentages. The relationship between the LNY and recurrence was assessed using the Man-Whitney U test. Furthermore, the study cohort was categorized into 2 groups based on the median LNY for further analysis. Baseline variables related to the LNY and recurrence rate were analyzed using Man Whitney or Fischer exact test. A $p$-value of $<0.05$ was considered statistically significant.

Results. Our study cohort comprised of 40 (male=27; females=13) cN0 patients who underwent treatment for histologically diagnosed OCSCC. Patients belonged to the age range of 34 years to 86 years with a mean age of $60.08+/-13.153$ years. Tongue (55\%) was the commonly affected site in the oral cavity. Based on the TNM classification, $42.5 \%$ of patients were categorized as TNM stage II followed by stage IV (27.5\%). Tumor-node-metastasis staging was unknown in $1(2.5 \%)$ patient. All patients underwent surgical resection of the tumor along with SND of level I to level III nodes. Nineteen (47.5\%) patients further received adjuvant therapies. The mean LNY in our study was $38.65 \pm 25.41$ (range 7-98). We observed recurrence only in $4(10 \%)$ patients. Details of the demographic characteristics, site of tumor, diagnosis, treatment and its outcome are summarized in Table 1.

There was no significant difference between the number of LNs retrieved (LNY) during surgery and the recurrence rate $(p=0.892)$ (Table 2$)$. Factors influencing the LNY and recurrence of OCSCC are summarized in Table 3. There was no significant association between LNY with age $(p=0.121)$, gender $(p=0.209)$, site $(p=0.519)$, tumor size $(p=0.416)$, and treatment type
Table 1 - Frequency distribution of study variables.

\begin{tabular}{|c|c|c|}
\hline Variables & $\mathbf{n}$ & $\%$ \\
\hline \multicolumn{3}{|l|}{ Gender } \\
\hline Male & 27 & 67.5 \\
\hline Female & 13 & 32.5 \\
\hline \multicolumn{3}{|l|}{ Sub-site } \\
\hline Buccal mucosa & 4 & 10.0 \\
\hline Tongue & 22 & 55.0 \\
\hline Floor of the mouth & 3 & 7.5 \\
\hline Alveolus/mandible & 4 & 10.0 \\
\hline Retromolar trigone & 3 & 7.5 \\
\hline Lower lip & 1 & 2.5 \\
\hline Hard palate & 2 & 5.0 \\
\hline Gingiva & 1 & 2.5 \\
\hline \multicolumn{3}{|l|}{ Histological code } \\
\hline $\begin{array}{l}\text { Moderately differentiated } \\
\text { squamous cell carcinoma }\end{array}$ & 3 & 7.5 \\
\hline SCC & 37 & 92.5 \\
\hline \multicolumn{3}{|l|}{ TNM staging } \\
\hline Stage I & 9 & 22.5 \\
\hline Stage II & 17 & 42.5 \\
\hline Stage III & 2 & 5.0 \\
\hline Stage IV & 11 & 27.5 \\
\hline unknown & 1 & 2.5 \\
\hline \multicolumn{3}{|l|}{ Treatment } \\
\hline Surgery & 21 & 52.5 \\
\hline Surgery+ chemotherapy & 1 & 2.5 \\
\hline Surgery+ radiotherapy & 14 & 35.0 \\
\hline Surgery+ chemo - radiotherapy & 4 & 10.0 \\
\hline \multicolumn{3}{|l|}{ Recurrence } \\
\hline Yes & 4 & 10.0 \\
\hline No & 36 & 90.0 \\
\hline
\end{tabular}

(0.532). Similarly, age $(p=0.972)$, gender $(p=0.284)$, site $(p=0.920)$, tumor size $(p=0.830)$ and treatment type $(0.458)$ had no effect on the recurrence rate.

Discussion. Nodal involvement of the neck in OCSCC increases the risk of recurrence, and poor outcome. ${ }^{8}$ With the limited role of diagnostic imaging, SND with lymphadenectomy remains the gold standard for diagnosing occult metastatic disease and has been recommended as a treatment protocol for $\mathrm{cN} 0$ OCSCC patients. ${ }^{9}$ All patients in our study underwent surgical resection of the primary tumor and SND of supramylohyoid lymph nodes (level I to level III). The extent of neck dissection determines the number of dissected LNs and LNY, which influence the survival of head and neck cancer patients. ${ }^{10}$ Kuo et $\mathrm{al}^{6}$ reported a positive association between meticulous and extensive neck dissection and LNY. However, with no definitive 
Table 2 - Effect of lymph node yield on the treatment outcome.

\begin{tabular}{lccccccc}
\hline Recurrence & $\mathbf{n}$ & Median & Mean rank & Sum of ranks & Mann-Whitney U & $\mathrm{Z}$ & $P$ value \\
\hline Yes & 4 & 35.00 & 27.25 & 85.00 & \multirow{2}{*}{69.000} & -0.135 & 0.892 \\
No & 36 & 29.00 & 20.42 & 735.00 & & & \\
\hline
\end{tabular}

Table 3 - Factors influencing the lymph node yield and recurrence rates.

\begin{tabular}{|c|c|c|c|c|c|c|}
\hline Variable & $<31 \mathrm{LNY}$ & $>31 \mathrm{LNY}$ & $P$-value & Recurrence present & Recurrence absent & $P$-value \\
\hline Age (in years) (mean+ $S D)$ & $63 \pm 13.77$ & $56.5 \pm 11.73$ & 0.121 & $60.33 \pm 9.504$ & $60.05 \pm 13.505$ & 0.972 \\
\hline \multicolumn{7}{|l|}{ Gender } \\
\hline Male & 13 & 14 & \multirow{2}{*}{0.209} & 4 & 23 & \multirow[t]{2}{*}{0.284} \\
\hline Female & 9 & 4 & & 0 & 13 & \\
\hline \multicolumn{7}{|l|}{ Sites } \\
\hline Buccal mucosa & 2 & 2 & \multirow{8}{*}{0.519} & 1 & 3 & \multirow{8}{*}{0.920} \\
\hline Tongue & 12 & 10 & & 3 & 19 & \\
\hline Floor of mouth & 1 & 2 & & 0 & 3 & \\
\hline Alveolus/mandible & 1 & 3 & & 0 & 4 & \\
\hline Retromolar trigone & 3 & 0 & & 0 & 3 & \\
\hline Lower lip & 1 & 0 & & 0 & 1 & \\
\hline Hard palate & 1 & 1 & & 0 & 2 & \\
\hline Gingiva & 1 & 0 & & 0 & 1 & \\
\hline \multicolumn{7}{|l|}{ Tstage } \\
\hline $\mathrm{T} 1$ & 6 & 3 & & 0 & 9 & \multirow{5}{*}{0.830} \\
\hline $\mathrm{T} 2$ & 11 & 6 & & 2 & 15 & \\
\hline T3 & 1 & 1 & 0.416 & 0 & 2 & \\
\hline $\mathrm{T} 4$ & 4 & 7 & & 2 & 9 & \\
\hline $\mathrm{T} 5$ & 0 & 1 & & 0 & 1 & \\
\hline \multicolumn{7}{|l|}{ Treatment } \\
\hline Surgery & 11 & 10 & \multirow{4}{*}{0.532} & 1 & 20 & \multirow{4}{*}{0.458} \\
\hline Surgery + chemotherapy & 0 & 1 & & 0 & 1 & \\
\hline Surgery + radiotherapy & 9 & 5 & & 3 & 11 & \\
\hline $\begin{array}{l}\text { Surgery }+ \\
\text { Chemoradiotherapy }\end{array}$ & 2 & 3 & & 0 & 4 & \\
\hline
\end{tabular}

guidelines, the decision on the extent of surgery is dependent on the surgeon's judgment, expertise, and decision-making from previous experience. In our study, the number of LN dissected ranged from 7 to 98 with a mean LNY of $38.65 \pm 25.41$, which is higher than reported by Muttagi et $\mathrm{al}^{11}(21.97 \pm 5.57)$ and Pou et $\mathrm{a}^{12}$ $(21.5 \pm 12.9)$. The difference in the mean LNY and wide range of nodal yield is attributed to the tumor site and lymphatic drainage, surgical technique used, the extent of dissection, and pathological examination.

In a meta-analysis of 10 OCSCC studies, de Kort et $\mathrm{al}^{13}$ conferred an improved overall survival of cancer patients associated with higher LNY. Higher LNY has advantages in the diagnostic, therapeutic front. Firstly, higher LNY acts as a diagnostic aid for the accurate pathological staging of the neck, which subsequently impacts the treatment plan thereafter. ${ }^{4,5}$ Secondly, higher LNY increases the likelihood of removing the occult metastatic disease. Finally, higher LNY reflects on the quality care of the surgeon and the institution, resulting in an improved outcome. ${ }^{6}$ On the other hand, decreased survival in patients with low LNY implicates the undertreatment of occult neck disease resulting in regional failure. Despite the known advantage of LNY, there is no consensus with the minimal LNY for OCSCC. While Bottcher et $\mathrm{al}^{14}$ reported decreased survival rate and increased risk of locoregional recurrence with $<18$ LNY, Kuo et $\mathrm{al}^{8}$ and Lemieux et $\mathrm{al}^{15}$ observed increased survival in cN0 patients with $>16$ LNY and $>21$ LNY, respectively. Arun et $\mathrm{al}^{5}$ did not observe a significant 
difference in risk of recurrence and improved outcome with a median cut-off of 36 nodes. In our study cohort, the overall LNY did not affect the recurrence rate.

Following the initial treatment, Chow et $\mathrm{al}^{9}$ reported $23.1 \%$ local recurrence, $12.8 \%$ regional recurrence and $7.7 \%$ distant metastasis in OCSCC patients. In our study, the recurrence was comparatively less, with a frequency of $10 \%$. Gender, oral sub-sites including oral tongue and floor of the mouth, TNM stage, increased tumor thickness, skin invasion, LNY, type of initial treatment, and postoperative adjuvant therapy are the prognostic indicators of oral cancer. ${ }^{2,5,9}$ In our study, recurrence was present only in male patients, of whom 3 patients had tongue cancer, and one had cancer in the buccal mucosa. Recurrence was noted in patients with stages T2 and T4 and 3 patients who underwent radiotherapy. However, there was no significant association between patient, tumor characteristics, adjuvant therapy and recurrence.

Factors such as patient's age, immunological response, primary tumor size, TNM stage, anatomic location, presence of oral potentially malignant disorder, prior history of radiation therapy, delay in surgery following diagnosis influence the LNY. Moreover, LN hypertrophy is influenced by many physiological and pathological factors, and it differs among individuals. ${ }^{10}$ More importantly, the extent of training and experience of the surgeon, surgical technique used, duration of formalin fixation and method of handling the specimen, the pathologist's experience in harvesting the LNs, different reporting techniques followed influence the overall LNY. ${ }^{13}$ Muttagi et $\mathrm{al}^{11}$ observed higher LNY in patients who delayed surgery after the initial diagnosis, suggesting disease progression during the waiting period. Also, the presence of oral potentially malignant disorder increased LNY. Increased LNY could be due to the inflammatory response to the non-healing ulcer and its related symptoms, including trismus and poor oral hygiene.

The findings of our study imply that a good neck dissection and thorough sampling of $\mathrm{LN}$ increases the prognosis of patients. Survival depends on the quality of dissection, rather than the quantity of LNY. Despite the absence of specific guidelines concerning the optimal number of sampled LNs, SND with a good quality will no doubt impact the treatment outcome and thus can be used as a tool to assess the quality of operative techniques and pathological examinations, which further provide insights on ways to improve the quality of care.

Study limitations. Our sample size was limited as we used only node-negative samples with different oral cavity subsites. The study's retrospective nature was another limitation, as the retrospective collection of information may have diverse and variable information due to the difference in the data collection methods used by different clinicians. Although all patients underwent supramylohyoid neck dissection as per the guidelines, we could not control the surgeon and pathologist counting the LNY and relied on the pathology reports. Histologic depth of invasion also plays a vital role in cancer recurrence, and the same was not considered.

Further large-scale prospective studies including multicentered data or nationwide databases are warranted to understand the implications of the extent of SND and LNY on the treatment outcome of OCSCC. Additionally, minimal LNY required for specific sub-sites of OCSCC also needs to be assessed due to variable lymphatic drainage, especially for the tumors involving the tongue and floor of the mouth, as they are more liable for LN metastasis. Furthermore, guidelines must be established for surgical dissection, minimal LNY needed to identify occult metastasis for individual sites of the oral cavity and different tumor stages, and pathological interpretation of neck samples.

In conclusion, there is no significant correlation between LNY and recurrence in cN0 OCSCC patients. The findings suggest that an LNY obtained from a meticulous neck dissection and a thorough pathologic sectioning and interpretation of samples are the basis for an adequate cancer staging. This further improves the quality of care and the clinical outcome. However, it is essential to develop specific guidelines to aid the surgeons and pathologists to identify an adequate number of LNs from the neck to minimize the risk of leaving the occult nodal metastasis.

Received 19th July 2021. Accepted 1st September 2021.

From the Department of Otolaryngology-Head and Neck Surgery (Altuwaijri Alessa, Aldhahri, Al-Qabtani), College of Medicine; from the Department of Surgery (Abuhaimed), King Saud University; and from the Department of Otorhinolaryngology Head and Neck Surgery (Altuwaijri, Bedaiwi, Almayouf, Albarrak, Al-Qahtani), King Fahad Medical City, Riyadh, Kingdom of Saudi Arabia.

Address correspondence and reprints request to: Dr. Ahmad A. Altuwaijri, Department of Otolaryngology-Head and Neck Surgery, College of Medicine, King Saud University, Riyadh, Kingdom of Saudi Arabia. Email: aamtu2@gmail.com ORCID ID : https://orcid.org/0000-0001-5649-9646

\section{References}

1. Alshehri BM. Trends in the incidence of oral cancer in Saudi Arabia from 1994 to 2015. World J Surg Oncol 2020; 18: 217.

2. Massey C, Dharmarajan A, Bannuru RR, Rebeiz E. Management of N0 neck in early oral squamous cell carcinoma: A systematic review and meta-analysis. Laryngoscope 2019; 129: E284-E298. 
3. de Bree R, Takes RP, Shah JP, Hamoir M, Kowalski LP, Robbins KT, et al. Elective neck dissection in oral squamous cell carcinoma: Past, present and future. Oral Oncol 2019; 90: 87-93.

4. Zenga J, Divi V, Stadler M, Massey B, Campbell B, Shukla $\mathrm{M}$, et al. Lymph node yield, depth of invasion, and survival in node-negative oral cavity cancer. Oral Oncol 2019; 98: 125-131.

5. Arun I, Maity N, Hameed S, Jain PV, Manikantan K, Sharan $\mathrm{R}$, et al. Lymph node characteristics and their prognostic significance in oral squamous cell carcinoma. Head Neck 2021; 43: 520-533.

6. Kuo P, Mehra S, Sosa JA, Roman SA, Husain ZA, Burtness BA, et al. Proposing prognostic thresholds for lymph node yield in clinically lymph node-negative and lymph node-positive cancers of the oral cavity. Cancer 2016; 122: 3624-3631.

7. Amin MB, Edge SB. AJCC Cancer Staging Manual. 8th ed. New York (NY): Springer; 2017.

8. Abdeyrim A, He S, Zhang Y, Mamtali G, Asla A, Yusup M, et al. Prognostic value of lymph node ratio in laryngeal and hypopharyngeal squamous cell carcinoma: a systematic review and meta-analysis. J Otolaryngol Head Neck Surg 2020; 49: 31.

9. Chow TL, Kwan WWY, Fung SC, Ho LI. Prognostic value of lymph node density in buccal squamous cell carcinoma. Am J Otolaryngol 2017; 38: 529-532.
10. Choi Y, Bin-Manie M, Roh JL, Cho KJ, Lee YS, Choi SH, et al. Metastatic lymph node burden predictive of survival in patients undergoing primary surgery for laryngeal and hypopharyngeal cancer. J Cancer Res Clin Oncol 2019; 145: 2565-2572.

11. Muttagi SS, Patil BR, Godhi AS, Arora DK, Hallikerimath $\mathrm{SR}$, Kale AD. Clinico-pathological factors affecting lymph node yield in Indian patients with locally advanced squamous cell carcinoma of mandibular Gingivo-Buccal sulcus. Indian J Cancer 2016; 53: 239-243.

12. Pou JD, Barton BM, Lawlor CM, Frederick CH, Moore BA, Hasney CP. Minimum lymph node yield in elective level I-III neck dissection. Laryngoscope 2017; 127: 2070-2073.

13. de Kort WWB, Maas SLN, Van Es RJJ, Willems SM. Prognostic value of the nodal yield in head and neck squamous cell carcinoma: A systematic review. Head Neck 2019; 41: 2801-2810.

14. Böttcher A, Dommerich S, Sander S, Olze H, Stromberger C, Coordes A, et al. Nodal yield of neck dissections and influence on outcome in laryngectomized patients. Eur Arch Otorhinolaryngol 2016; 273: 3321-3329.

15. Lemieux A, Kedarisetty S, Raju S, Orosco R, Coffey C. Lymph node yield as a predictor of survival in pathologically node negative oral cavity carcinoma. Otolaryngol Head Neck Surg 2016; 154: 465-472. 